\title{
EFECTOS DE UN PROGRAMA DE AQUAEROBIC SOBRE LA COMPOSICIÓN CORPORAL, EN LAS MUJERES ACTIVAS DE MEDIANA EDAD
}

\author{
Juan José Manzano, Christian Clemente, Asunción Grager y GuillermoOlcina \\ Facultad de Ciencias del Deporte. Universidad de Extremadura, España \\ manzanoventurajj@hotmail.com
}

RESUMEN

\begin{abstract}
Objetivo: Describir y analizar las modificaciones en la composición corporal, en las mujeres de mediana edad, después de realizar un programa de actividad física, basado en el aquaerobic, y un posterior periodo de desentrenamiento. Material y método: El grupo analizado estuvo integrado por diecisiete mujeres de Arroyo de San Serván (Badajoz), físicamente activas (habían realizado programas de gimnasia de mantenimiento, con una frecuencia de una a tres sesiones semanales, durante los últimos tres meses), con una edad media de 53,6 \pm 9,1 años. El programa se desarrolló con sesiones de sesenta minutos durante cinco días a la semana, por seis semanas, con un periodo de desentrenamiento de cuatro semanas. Se realizaron tres evaluaciones antropométricas: al iniciar el programa, al finalizar el mismo, tras el periodo de desentrenamiento. En cada medición, se determinaban la talla y el peso, seis pliegues cutáneos, cuatro perímetros corporales y tres diámetros óseos. El porcentaje de grasa fue obtenido a través de los datos arrojados en cuatro pliegues cutáneos (tricipital, subescapular, suprailíaco y abdominal) mediante la fórmula de Yuhasz, modificada por Faulkner (1968). Por su parte, el porcentaje muscular fue calculado, a partir del porcentaje de la masa total, menos el porcentaje óseo (Rocha, 1975), el porcentaje graso (Faulkner, 1968) y el porcentaje residual, a través de la ecuación de Wurch (Esparza, 1993), de los sujetos medidos, según la propuesta básica de Matiegka (modelo de los cuatro componentes). Resultados: después de realizar el programa, se observó un aumento significativo en el porcentaje muscular: $32.69 \pm 2.69 \%$ vs. $33.44 \pm 2.89 \%$ ( $<<0.05$ ), en el peso muscular: $23.45 \pm 3.53 \mathrm{~kg}$ vs. $24.02 \pm 3.33 \mathrm{~kg}(\mathrm{p}<0.05)$, y un descenso significativo del componente graso reflejado, mediante el sumatorio de pliegues cutáneos: $193.66 \pm 25.54 \mathrm{~mm}$ vs. $188.38 \pm 25.67 \mathrm{~mm}(\mathrm{P}<0,05)$, más específicamente, en el pliegue suprailíaco, $30.47 \pm 8.49 \mathrm{~mm}$ vs. $28.00 \pm 8.78 \mathrm{~mm}$ ( $\mathrm{p}<0,05)$ Estas tendencias se mantuvieron durante el periodo de desentrenamiento, en el porcentaje muscular: $33.44 \pm 2.89 \%$ vs. $34,25 \pm 3,04 \%$ (p $<0,01$ con respecto a la inicial), en el peso muscular: $24.02 \pm 3.33 \mathrm{~kg}$ vs $24,74 \pm 3,64 \mathrm{~kg}$ ( $\mathrm{p}<0,01$ con respecto a la inicial), el sumatorio de pliegues cutáneos: $188.38 \pm 25.67 \mathrm{~mm}$ vs $183,41 \pm 26,65 \mathrm{~mm}$ ( $\mathrm{p}<0,05$ con respecto a la inicial). Conclusiones: $\mathrm{La}$ participación en programas de aquaerobics de corta duración bajo las condiciones de este estudio provoca descensos del componente graso y aumentos significativos en el componente muscular, en las mujeres activas de mediana edad.
\end{abstract}

PALABRAS CLAVES: salud, porcentaje graso, IMC, porcentaje muscular, desentrenamiento.

\section{EFFECTS OF A WATER AEROBICS PROGRAM ON THE BODY COMPOSITION OF ACTIVE MIDDLE-AGED WOMEN}

\begin{abstract}
Objective: Describe and analyze changes in body composition of middle-aged women after participating in a water aerobics physical activity program and a subsequent detraining period. Materials and Methods: The group analyzed consisted of 17 women from Arroyo de San Serván (Badajoz), who were physically active (had participated in fitness gymnastics programs one to three sessions per week for the past three months), with an average age of $53.6 \pm 9.1$ years. The program was comprised of sixty minute-sessions five days a week, for 6 weeks, with a 4-week detraining period. Three anthropometric evaluations were conducted: at the beginning and at the end of the program and after the detraining period. Size and weight were recorded during each measurement, including six skinfolds, four body circumferences and three bone diameters. Fat percentage was obtained from four skinfolds (triceps, subscapular, suprailiac and abdominal) using the Yuhasz formula modified by Faulkner (1968). The muscle percentage was calculated from total mass percentage less bone percentage (Rocha, 1975), body fat percentage (Faulkner, 1968) and the residual percentage using the Wurch's equation (Esparza, 1993) of subjects measured, based on Matiegka's basic proposal (four components model). Results: after the program a significant increase was observed in muscle percentage: $32.69 \pm 2.69 \%$ vs. $33.44 \pm 2.89 \%$ (p <0.05), in muscle weight: $23.45 \pm 3.53 \mathrm{~kg}$ vs. $24.02 \pm 3.33 \mathrm{~kg}$ (p $<0.05$ ), as well as a significant decrease in the fat component reflected in the sum of skinfolds: $193.66 \pm 25.54 \mathrm{~mm}$ vs. $188.38 \pm 25.67 \mathrm{~mm}(\mathrm{p}<0.05)$ and more specifically in the suprailiac skinfold, $30.47 \pm 8.49 \mathrm{~mm}$. vs $28.00 \pm 8.78 \mathrm{~mm}$ $(\mathrm{p}<0.05)$. These trends were maintained during the detraining period, specifically in muscle percentage: $33.44 \pm$ $2.89 \%$ vs. $34.25 \pm 3.04 \%$ ( $p<0.01$ compared to initial measurement), muscle weight: $24.02 \pm 3.33 \mathrm{~kg}$ vs $24.74 \pm 3.64$ $\mathrm{kg}$ ( $\mathrm{p}<0.01$ compared to initial measurement), the sum of skinfolds: $188.38 \pm 25.67 \mathrm{~mm}$ vs $183.41 \pm 26.65 \mathrm{~mm}$ (p $<0.05$ compared to initial measurement). Conclusions: Participating in water aerobics programs for a short period of time under the conditions of this study decreases the fat component and significantly increases the muscle component in active middle-aged women.
\end{abstract}




\section{INTRODUCCIÓN}

En la actualidad, existe una consideración social sobre el ejercicio físico, como un medio para mantener o mejorar la salud y calidad de vida. De este modo, se refleja en la Encuesta Europea de Salud en España (Ministerio de Sanidad Política Social e Igualdad. Instituto nacional de estadística, 2009). Lo anterior se debe, por una parte, al conocimiento que se ha ido adquiriendo en los últimos años, sobre los efectos saludables de la práctica continuada y sistematizada de la actividad motriz, en el nivel físico (Tabernero, Villa, Márquez y García, 2000; Castillo, Ortega y Ruiz, 2005), psicológico (González, Núñez y Salvador, 1997; Huertas et al., 2003; Morilla, 2001) y social (Aparicio, 2010), y por otra parte, al concepto del deporte para todos (Consejo de Europa, 1975), que ha permitido la incorporación a la práctica física, de grupos de población, como el de la mujer, el cual en la actualidad, se encuentra en continuo crecimiento, en cuanto a los hábitos mencionados se refiere (García, 2006).

A raíz de esta situación, han surgido multitud de programas grupales con diversas características (aeróbicas, tonificantes y relajantes), donde son asiduas las mujeres de mediana edad (cuarenta y cinco a sesenta y cinco años) en su gran mayoría (García, 2006; Jiménez-Beatty et al., 2008; Martínez et al., 2009). No obstante, esta gama de actividades grupales es muy variada, siendo necesario, por lo tanto, profundizar en los conocimientos sobre los distintos efectos que pueden tener sobre la salud y la calidad de vida, en cada una de ellas.

En este sentido, nuestro estudio se va a centrar en analizar los efectos, en la población femenina adulta, de la actividad física realizada en el medio acuático, principalmente, nos centraremos en los efectos antropométricos.

En investigaciones relacionadas con la condición física, se concluye que la actividad física acuática mejora la capacidad cardiorrespiratoria, en las mujeres adultas y mayores (Nikolai, Novotny, Bohnen, Schleis y Dalleck, 2009). Además, se sabe que en las mujeres jóvenes sanas, después de un programa de cuatro semanas, mejoran la mayoría de los parámetros de condición física, relacionados con la salud, en especial, los vinculados con la fuerza, la flexibilidad y el equilibrio (Saavedra, Escalante, Pino, De la Cruz y Rodríguez, 2006).

En relación con la composición corporal, se han realizado investigaciones con ocho meses de duración, donde se encuentran descensos significativos del porcentaje corporal graso, en las mujeres premenopáusicas sedentarias (Saavedra, De la Cruz, Escalante y Rodríguez, 2007). También, existen estudios de seis meses de duración, donde se reflejan descensos en el peso y el IMC, aunque no de forma significativas, tanto para las mujeres como para los hombres adultos sedentarios (López et al., 2005).

La mayoría de los estudios referentes con el análisis antropométrico en programas de aquaerobic, data de intervenciones trimestrales (Bocalini, Serra, Murad y Levy, 2008; Gappmaier, Lake, Nelson y Fisher, 2006; Greene, Lake, Nelson, y Fisher, 2009). Para este periodo, se observó un mantenimiento del peso corporal en las mujeres postmenopáusicas sedentarias, cuando realizaron ejercicios con intensidades del $70 \%$ de la frecuencia cardiaca máxima (Bocalini et al., 2008), descensos significativos en el peso corporal, el IMC, el porcentaje graso y la masa grasa, así como aumentos significativos en la masa magra de las extremidades inferiores, en los sujetos obesos sedentarios, quienes realizaban tres sesiones semanales (Greene et al., 2009), los mismos efectos que cuando se acompañaba la actividad con dieta (Gappmaier et al., 2006). Con una duración inferior, con cuatro semanas de práctica de aquaerobic, se ha detectado un descenso significativo del porcentaje graso, en las mujeres jóvenes semiactivas (Saavedra et al., 2006). 
Como podemos observar, existen muy pocos estudios que analicen los efectos del aquaerobic en la población femenina adulta, con intervenciones de una duración inferior a dos meses. A la escasez de estos estudios, se suma el desconocimiento de los efectos producidos por el aquaerobic, en el nivel antropométrico, en los sujetos activos, pues la mayoría de los estudios, se centran en las personas sedentarias, con sobrepeso elevado u obesidad, como hemos visto anteriormente. Por otro lado, no hay investigaciones que reflejen la duración de esos efectos, cuando no se mantiene la actividad.

De acuerdo con lo mencionado anteriormente, el objetivo del presente estudio consiste en contribuir, a ampliar el conocimiento de los efectos saludables de la práctica del aquaerobic, centrándonos en el análisis de los cambios antropométricos, producidos en las mujeres activas de mediana edad, en un programa de corta duración, así también, comprobar la duración de los mismos, tras un periodo de desentrenamiento.

\section{METODOLOGIA}

\section{Sujetos}

Los sujetos participantes en el estudio fue un grupo de diecisiete mujeres, seleccionadas de forma aleatoria, de las inscritas en un programa de actividad física de aquaerobic, siempre, que cumplieran los criterios de inclusión siguientes: mujeres mayores de cuarenta años, ubicadas en la localidad de Arroyo de San Serván (Badajoz), sin ninguna lesión o patología, practicantes de gimnasia de mantenimiento, en un periodo superior de tres meses, anterior al programa, con una asistencia de más del $85 \%$ a las sesiones del programa; que no realizaran ninguna otra actividad física programada.

El grupo seleccionado contaba con una edad media de 53,6 $\pm 9,1$ años.

\section{Procedimiento}

Antes de iniciar el programa de intervención, se realizó una entrevista personalizada a los sujetos de estudio, con el objetivo de incluir en el programa a las usuarias, quienes cumplieran con los criterios del punto anterior.

La toma de los datos antropométricos, para efectuar el estudio de la composición corporal, mediante métodos cineantropométricos, se realizó en tres momentos diferenciados: al comienzo del programa, con el fin de caracterizar la muestra y determinar los valores iniciales, al finalizar el programa, para analizar el efecto del mismo, tras el periodo de desentrenamiento de cuatro semanas, para estudiar su efecto y la durabilidad de los cambios.

\section{Recolección de los datos}

Los procedimientos y los controles en los sujetos han sido realizados, tras la obtención de un consentimiento informado. Las diferentes medidas antropométricas realizadas en el estudio fueron las siguientes:

El Peso y la talla: Los mismos se determinaron, según el manual I.S.A.K (normas establecidas por la sociedad internacional para el desarrollo de la cineantropometría, Marfell-Jones, Olds, Stewart, and Carter, 2006).

Los pliegues cutáneos: para obtener la medición de los pliegues cutáneos, se utilizó el protocolo I.S.A.K. (Marfell-Jones et al., 2006). Los pliegues medidos fueron el tricipital, pliegue subescapular, pliegue abdominal, pliegue suprailíaco, pliegue del muslo y pliegue de la pierna.

Los perímetros corporales: con el fin de adquirir las medidas de los perímetros corporales, se establecieron las mediciones, según marca el protocolo de la Sociedad Internacional de la 
Cineantropometría I.S.A.K. (Marfell-Jones et al., 2006). Se determinaron los perímetros de la cintura, de la cadera, del brazo relazado y de la pierna relajada.

Los diámetros óseos: para conseguir los tres diámetros óseos, se establecieron las mediciones, según el protocolo I.S.A.K. (Marfell-Jones et al., 2006). Los diámetros óseos medidos fueron el biestiloideo, el bicondiloideo del codo y el bicondiloideo del fémur.

A continuación, describimos los instrumentos de medición utilizados, tendientes a obtener los datos:

Para obtener la talla y el peso, se utilizó una báscula digital de columna Seca 703 con tallímetro. Los pliegues cutáneos fueron obtenidos a través de un plicómetro Holten, con zona de medidas 0 - $48 \mathrm{~mm}$., graduación $0,2 \mathrm{~mm}$. y una presión constante $10 \mathrm{gr} / \mathrm{mm} 2$. Los diámetros corporales fueron adquiridos mediante una cinta métrica, con el fin de conseguir las medidas de los diámetros óseos, se utilizó un paquímetro Holtein con una zona de medida 0 -140 mm.

Tras el registro de todas las medidas, se realizaron una serie de cálculos, orientados a obtener de cada sujeto, el índice de la masa corporal (Quetelet), el índice cintura-cadera (ICC), el sumatorio de los pliegues cutáneos, el peso graso y el porcentaje de grasa (Yuhasz, modificada por Faulkner, 1968)

$$
\% \text { Graso }=\Sigma 4 \text { plg }(\text { t se si a }) \times 0.153+5.783
$$

siendo: t: tríceps; se: Subescapular; si: Suprailíaco; a: Abdominal.

El peso muscular y el porcentaje muscular (Lee et al., 2000), el peso óseo y el porcentaje óseo (Rocha, 1975), así como el peso residual, según la ecuación de Wurch.

\section{Programa de intervención}

El programa de aquaerobic tuvo lugar en una piscina de vaso olímpico $(50 \times 25 \mathrm{~m})$, con una profundidad de $1,40 \mathrm{~m}$, con el agua con una temperatura de unos $22-25^{\circ} \mathrm{C}$. La duración del programa fue de un total de seis semanas, realizando cinco sesiones semanales con una duración de sesenta minutos en cada una de ellas.

Todas las estructuras de las sesiones fueron similares, consistió en lo siguiente: Calentamiento, con una duración de cinco a diez minutos, donde se incluía la movilidad articular y el estiramiento fuera del agua.

Parte principal, desarrollada en el agua, con una duración entre cuarenta y cincuenta minutos. El trabajo desarrollado era fundamentalmente aeróbico, realizado al $60 \%$ de la frecuencia cardíaca máxima teórica (220-edad). En todas las sesiones, se trabajaron siempre, las siguientes zonas corporales: piernas, brazos, caderas, abdominales y glúteos. Por último, vuelta a la calma, desarrollada en los últimos cinco minutos de la sesión, su actividad principal consistió en los estiramientos con acompañamiento musical.

\section{Análisis estadístico}

Se realizó la prueba de Kolmorov-Smirnov, dirigida a comparar la normalidad de los datos; y el Test de Levene, con el fin de asegurar la homogeneidad de la varianza. Puesto que las variables analizadas cumplían con los criterios de normalidad y de homogeneidad de la varianza, se procedió a un análisis estadístico de los datos, mediante las pruebas paramétricas.

Se utilizó un Modelo Lineal General de Medidas Repetidas, para estudiar los cambios ocurridos en las diferentes variables estudiadas, como consecuencia del programa de ejercicio físico, comparando los valores iniciales con los valores finales, obtenidos después del programa y tras el periodo del desentrenamiento. Se utilizó la prueba post-hoc de Bonferroni, para estudiar estas diferencias. 
URL www.una.ac.cr/mhsalud

Los resultados fueron expresados como la media \pm desviación estándar. Se consideraron como estadísticamente significativas, aquellas diferencias que alcanzaron un valor de $p<0,05$. Estos valores se indican en tres niveles, según la significatividad, $\mathrm{p}<0.05, \mathrm{p}<0.01$ y $\mathrm{p}<0.001$.

El tratamiento estadístico se realizó mediante el software informático SPSS versión 16.0, para Windows.

\section{RESULTADOS}

En la tabla 1, se muestran los datos generales de los sujetos. Se observa cómo se mantienen el peso corporal y el IMC en el momento de finalizar el programa, y un mes después de la conclusión del mismo. En cuanto al componente residual, la ecuación de Wurch (Esparza, 1993) siempre, estima el mismo porcentaje para este grupo de población.

\section{Tabla 1.}

Datos generales de los sujetos quienes recibieron la intervención

\begin{tabular}{lccc}
\hline & Inicial & Final & Desentrenamiento \\
\hline Peso $(\mathbf{k g})$ & $72,18 \pm 12,43$ & $72,39 \pm 11,96$ & $72,76 \pm 12,26$ \\
IMC $\left(\mathbf{k g} / \mathbf{m}^{\mathbf{2}}\right)$ & $29,56 \pm 5,21$ & $29,64 \pm 5,06$ & $29,78 \pm 5,11$ \\
Peso óseo $(\mathbf{k g})$ & $10,40 \pm 1,14$ & $10,40 \pm 1,14$ & $10,40 \pm 1,14$ \\
\% Residual & $20,9 \pm 0$ & $20,9 \pm 0$ & $20,9 \pm 0$ \\
\hline
\end{tabular}

En la tabla 2, se reflejan los datos, en relación con el componente graso de los sujetos. Se observa un descenso en el porcentaje graso al concluir el programa y un descenso significativo $(p<0,02)$, tras un mes de desentrenamiento, acompañados de un ligero descenso en el peso graso. La suma total de pliegues cutáneos fue menor, tanto a la finalización del programa de intervención ( $p<0,04)$, como tras el periodo de desentrenamiento $(\mathrm{p}<0,022)$. El pliegue más sensible a los cambios, fue el pliegue suprailiaco, con un descenso estadísticamente significativo, tanto al final del programa $(\mathrm{p}<0,047)$, como tras el periodo de desentrenamiento $(\mathrm{p}<0,01)$. No se observan cambios significativos en el resto de pliegues cutáneos, en los perímetros de la cintura o de la cadera, ni en el ICC a lo largo del estudio.

\section{Tabla 2.}

Componente graso de los sujetos que recibieron la intervención

\begin{tabular}{lccc}
\hline & Inicial & Final & Desentrenamiento \\
\hline \% graso & $32,22 \pm 3,65$ & $31,54 \pm 3,72$ & $30,78 \pm 3,82^{+}$ \\
Peso graso (kg) & $23,59 \pm 6,07$ & $23,16 \pm 5,96$ & $22,73 \pm 6,01$ \\
$\sum$ Pliegues (mm) & $193,66 \pm 25,54$ & $188,38 \pm 25,67^{*}$ & $183,41 \pm 26,65^{+}$ \\
Pliegue abdominal (mm) & $40,97 \pm 6,88$ & $41,84 \pm 5,81$ & $41,56 \pm 6,84$ \\
Pliegue suprailíaco (mm) & $30,47 \pm 8,49$ & $28,00 \pm 8,78^{*}$ & $27,53 \pm 8,47^{++}$ \\
Pliegue subescapular (mm) & $20,51 \pm 6,85$ & $20,72 \pm 6,24$ & $20,38 \pm 6,90$ \\
Pliegue tricipital (mm) & $32,25 \pm 3,89$ & $30,59 \pm 3,54$ & $30,50 \pm 4,43$ \\
Pliegue muslo (mm) & $42,31 \pm 3,51$ & $41,19 \pm 4,78$ & $40,75 \pm 4,94$ \\
Pliegue pierna (mm) & $26,56 \pm 7,62$ & $26,06 \pm 6,17$ & $25,22 \pm 5,37$ \\
ICC & $0,90 \pm 0,04$ & $0,90 \pm 0,03$ & $0,90 \pm 0,04$ \\
Ø Cintura (cm) & $93,59 \pm 14,43$ & $93,69 \pm 12,79$ & $93,63 \pm 13,15$ \\
$\emptyset$ Cadera (cm) & $103,16 \pm 13,20$ & $97,88 \pm 24,64$ & $103,75 \pm 13,41$ \\
\hline
\end{tabular}

${ }^{*} \mathrm{p}<0,05$ (inicial-final); ${ }^{+} \mathrm{P}<0,05$ (inicial-desentrenamiento) $++\mathrm{P}<0,01$ (inicial-desentrenamiento) 
En la tabla 3, se muestran los datos, en relación con la masa muscular de los sujetos, quienes recibieron la intervención. Se observa un incremento significativo en el porcentaje muscular $(p<0,035)$ y el peso muscular, tanto al finalizar el programa $(\mathrm{p}<0,043)$, como después del periodo del desentrenamiento ( $\mathrm{p}<0,008$ y $\mathrm{p}<0,02$, respectivamente), con respecto a los datos iniciales. También, se observa un ligero aumento en los perímetros del brazo y la pierna a lo largo del estudio, aunque no se alcanzó la significación estadística.

Tabla 3.

Componente muscular de los sujetos que recibieron la intervención.

\begin{tabular}{lccc}
\hline & INICIAL & FINAL & DESENTRENO \\
\hline \% Muscular & $32,69 \pm 2,69$ & $33,44 \pm 2,89^{*}$ & $34,25 \pm 3,04^{++}$ \\
Peso muscular (kg) & $23,45 \pm 3,53$ & $24,02 \pm 3,33^{*}$ & $24,74 \pm 3,64^{++}$ \\
Ø Brazo relajado (cm) & $29,19 \pm 2,94$ & $30,03 \pm 2,55$ & $30,44 \pm 3,50$ \\
Ø Pierna relajada $(\mathbf{c m})$ & $35,81 \pm 2,82$ & $36,44 \pm 3,08$ & $37,13 \pm 3,05$ \\
\hline
\end{tabular}

$* \mathrm{p}<0,05$ (inicial-final); ++ $\mathrm{P}<0,01$ (inicial-desentrenamiento)

\section{Discusión}

Según los resultados obtenidos en este estudio, se observan cambios significativos en el sumatorio de los pliegues corporales, una vez finalizado el programa de actividad física, aunque no existen cambios significativos, ni en el peso ni en el porcentaje graso. El factor que podría explicar la existencia de los cambios significativos en el sumatorio de pliegues y no, en el porcentaje graso, podría ser el número de pliegues contemplados en cada uno de los índices, pues para el sumatorio de los pliegues, se tienen en cuenta seis pliegues, mientras que para el cálculo del porcentaje graso, se tienen en cuenta cuatro.

Por su parte, sí existen cambios significativos en el componente muscular a la finalización del programa de aquaerobic, con respecto a los valores iniciales, aumentando tanto el porcentaje muscular ( $\mathrm{p}<0,035)$, como el peso muscular $(\mathrm{p}<0,043)$. Un mes después de concluir la intervención, estos valores siguen la tendencia marcada, a la finalización del programa de aquaerobic. De esta manera, comparando los valores iniciales con los del desentrenamiento, existe un descenso significativo, en el sumatorio de los pliegues cutáneos $(\mathrm{p}<0,022)$, apareciendo un descenso significativo en el porcentaje graso $(p<0,020)$. En esta línea, se observa un aumento, tanto en el peso muscular ( $p<0,02)$, como en el porcentaje muscular $(\mathrm{p}<0,008)$. No se observan cambios significativos, entre los valores finales y los del desentrenamiento, en los componentes corporales.

Al concluir el programa, los datos finales con respecto a los iniciales, no muestran cambios significativos en el componente graso, hablaríamos únicamente, de tendencia descentente, ya que sí hubo un cambio significativo en el sumatorio de los pliegues cutáneos. Esto es contrario a otros estudios, como el de Greene et al. (2009), donde se observan cambios significativos en el porcentaje graso y en la masa grasa, aunque el periodo de intervención es mayor (tres meses). Datos similares muestra el estudio de Gapmaier et al. (2006), siendo acompañado, en este caso, el programa de aquaerobic, con dieta hipocalórica. Uno de los estudios que más se acerca a nuestra intervención temporal, es el realizado por Saavedra et al. (2006), con cuatro semanas de intervención, ocho sesiones, en total de treinta minutos cada una, donde se observa un descenso significativo del porcentaje graso, en las mujeres jóvenes que no practicaban ningún deporte.

Las diferencias de los efectos producidas en los estudios mencionados, con los ocurridos en nuestra intervención, podríamos estimarlas principalmente, en la mayor duración temporal de éstos, y por otra parte, debido a las características de la muestra. Mientras que en los estudios mencionados, la muestra la conformaron mujeres pasivas, nuestro estudio se desarrolló con mujeres activas, según los 
principios del entrenamiento, los cambios son menores, por existir un menor rango de mejora (García, Ruiz y Navarro, 1996).

Aunque como hemos visto, no se producen cambios significativos, en el componente graso, en los valores finales del programa, sí se producen en el sumatorio de los pliegues. Dentro de los pliegues medidos, se puede observar cómo es el pliegue suprailíaco, el que desciende significativamente. En los restantes cinco pliegues, se producen cambios, sin embargo, no son significativos. No hay estudios que se relacionen en este sentido, con las actividades de aquaerobic, aunque estos resultados no coinciden con los datos reflejados por otros estudios. En este caso, después de tener en cuenta, varias regiones corporales y tras un programa de danza aeróbica y otro de jogging y ciclismo, en las mujeres de mediana edad, los cambios en el descenso graso se dan en las regiones del pliegue tricipital y subescapular, en una intervención trimestral (Shimamoto, Adachi, Takahashi, y Tanaka, 1998).

Sigue la misma línea, el estudio de Nild et al. (2000), donde después de un programa de fuerzaresistencia de seis meses, para las mujeres sedentarias, el mayor descenso graso se refleja en las extremidades superiores, con descensos del 31\%. Por otra parte, otros estudios referidos a la distribución de la grasa después de programas de actividad física, revelan otras zonas como las más proclives a la pérdida de grasa, como las piernas (Nild et al., 2000) y la zona abdominal (Irwin et al., 2003; Park et al., 2003), al finalizar sendos programas aeróbicos (fuerza-resistencia, saltos, bailes, caminatas, etc.) de doce y seis meses respectivamente, en las mujeres premenopáusicas obesas. Como vemos, cambian las zonas con mayor pérdida de grasa, en función de las distintas actividades físicas, de la duración de la intervención y de las características de la muestra.

También, resulta contradictorio el mantenimiento de los perímetros de la cintura y la cadera, en los sujetos de nuestros estudio, pues según reflejan los estudios revisados, después de programas de actividad física, se refleja un descenso en los perímetros mencionados (Shimamoto et al., 1998; Nildl et al., 2000; Jakicic, Marcus, Gallagher, Napolitano \& Lang, 2003; Irwin et al., 2003; Church et al., 2009), aunque en programas de una corta duración (cuatro semanas), se dan cambios en el perímetro de la cintura, pero, no en el de la cadera (Saavedra et al., 2006). Estas diferencias vienen dadas fundamentalmente, por las características de nuestra muestra, pues son sujetos activos con menor rango de mejora y se encuentran en la etapa peri menopáusica o menopáusica. Se ha demostrado que en este periodo, hay mayor resistencia a la pérdida de grasa, en las zonas centrales del cuerpo (Ley, Lees y Stevenson, 1992).

En relación con el componente muscular, se observan como el peso muscular y el porcentaje muscular aumentan significativamente, ( $\mathrm{p}<0,043$ y $\mathrm{p}<0,035$ respectivamente) a la conclusión del programa, con respecto a los datos iniciales. En los programas aeróbicos acuáticos, existe una tendencia en ese sentido. Así, en el estudio de Greene et al. (2009), se observa un aumento significativo de la masa muscular en las extremidades inferiores, después de doce semanas de entrenamiento, con un tapiz rodante con resistencia acuática, en los sujetos con sobrepeso.

Del mismo modo, en los programas de actividad física general, donde se destina parte del programa al trabajo de fuerza resistencia, o poseen intensidades altas aeróbicas, también, se observan incrementos significativos en la masa magra (Park, 2003), por otro lado, muy parecidos a las características de nuestro programa. En este sentido, se refleja una mejora significativa en el componente magro, después de seis meses de entrenamiento de fuerza-resistencia, en las mujeres obesas de mediana edad. En la misma línea, quedan reflejados los estudios de González, Delgado, Contreras y Vaquero (2003) y Kanemaru et al. (2010), aunque son programas específicos de fuerza. En este sentido, podemos afirmar, que en programas de actividad física de una intensidad media-alta, ya sean de corta o larga duración, hay una mejora en el componente muscular.

En cuanto al peso corporal y el IMC, no hubo cambios significativos de los datos finales a los iniciales, en la intervención que llevamos a cabo. Teniendo en cuenta, sólo los estudios relacionados con los programas de aquaerobic, se muestra como en los programas de corta duración, no se observan cambios en el peso, aún habiéndolos en la grasa corporal (Saavedra et al., 2006; Bocalini et al., 2003), 
debido a que aumenta también la masa magra. Por otra parte, en los programas con mayor duración temporal, se observa un descenso significativo del peso (Green et al., 2009; Gappmaier et al., 2006; López et al., 2005), pues la masa magra se estabiliza y la masa grasa sigue descendiendo, ya que los sujetos poseen condiciones de obesidad. Se justifica de la misma manera, el mantenimiento del índice de masa corporal, durante todo el programa, pues este índice depende tanto de la masa grasa, como de la masa magra.

Por lo tanto, en este sentido, la inexistencia de los cambios significativos en el peso y el IMC en nuestra intervención, se estima que podría ser, debida a la corta duración del programa de aquaerobic, que como hemos dicho antes, no facilitaría la pérdida de grasa de forma significativa.

Centrándonos en los datos que se muestran, tras un periodo de un mes de desentrenamiento, una vez finalizado el programa de aquaerobic, y analizando estos datos, tanto con los del inicio del programa, como con los finales, se observa que no ha habido ningún cambio entre los datos finales y los de desentrenamiento, no obstante, se han dado cambios significativos, entre los iniciales y los del desentrenamiento, concretamente, se ha seguido la tendencia de cambios significativos mostrados, entre los datos iniciales y los finales. Así, podemos observar, cambios sobre el componente muscular (porcentaje muscular y peso muscular) y sobre el componente graso (porcentaje graso y sumatorio de pliegues cutáneos), algo que no ocurría, entre los datos iniciales y los finales. Los restantes datos ofrecen cambios pero no significativos.

No existen referencias en cuanto al desentrenamiento, referentes a los programas de aquaerobic y composición corporal, por lo tanto, vamos a referenciar otros programas de actividad física, con la cautela que ello debe generar en el análisis. En general, todas las investigaciones analizadas muestran claros empeoramientos, en los valores obtenidos después del periodo del desentrenamiento (Giada, 1995; Prestes, De Lima, Frollini, Donatto, y Conte, 2009; Winters, 2000). De este modo, existen estudios donde se refleja un aumento del IMC, y cambios de composición corporal, con aumento en la grasa corporal, después de dos meses de desentreno en hombres adultos, quienes habían realizado actividad aeróbica de ciclismo y atletismo, durante seis meses (Giada et al., 1995).

Por otra parte, se observa un mantenimiento de la masa magra, después de una semana del desentrenamiento de un programa de doce semanas, orientado a la fuerza, en las mujeres adultas de veinte a veinticinco años (Prestes et al., 2009). Del mismo modo, se observa que para un grupo de mujeres premenopáusicas, se refleja un descenso significativo de la masa magra, después de seis meses del desentrenamiento de un programa, orientado a la fuerza de un año (Winters y Snow, 2000).

Como se puede observar, los periodos en el desentrenamiento referenciados, cuentan con una duración mínima superior a ocho semanas, muy alejados, de las cuatro de la intervención realizada. Se añade por otra parte, que el programa realizado se administra a una muestra de mujeres activas. Por lo tanto, los resultados obtenidos en nuestro estudio, se pueden explicar sobre dos líneas, la teoría de la supercompensación, mejoras obtenidas tras un periodo del descenso de la intensidad en la actividad física, o bien, debido al poco espacio temporal, desde la finalización del programa de intervención, siendo éste insuficiente, para denotar síntomas del desentrenamiento. En cualquier caso, no se puede afirmar, que los resultados obtenidos con respecto a la modificación de la composición corporal, son duraderos en un periodo de cuatro semanas, después del término del programa de intervención de aquaerobic, en mujeres activas de mediana edad, pues existen multitud de variables que pueden haber propiciado estos datos. No se ha controlado en ningún momento, la alimentación, la actividad física posterior al programa o el componente personal de los sujetos.

De acuerdo con lo mencionado en los párrafos anteriores, y con los resultados mostrados en los estudios que hemos referenciado, con descensos significativos en todos los componentes corporales, cuando se deja de practicar ejercicio físico, es necesario pensar en factores de práctica física, nutricionales o en un factor temporal, tendiente a interpretar adecuadamente, estos resultados indicados para próximos estudios. 


\section{CONCLUSION}

Tomando en cuenta, los resultados obtenidos en este estudio, se puede concluir que, las mujeres activas de mediana edad, participantes en los programas de aquaerobic de corta duración, practicando cinco días a la semana durante una hora, podrían obtener una ganancia de masa muscular, aunque podrían no obtener resultados, en cuanto al descenso del componente graso, así como a los descensos en el peso corporal y el IMC.

\section{BIBLIOGRAFÍA}

Aparicio, V., Carbonell-Baeza, A., Delgado-Fernández, M. (2010). Beneficios de la actividad física en personas mayores. Revista Internacional de Medicina y Ciencia de la Actividad Física y el Deporte, 10(40), 556-576.

Bocalini, D.S., Serra, A.J., Murad, N. \& Levy, R.F. (2008). Water- versus land-based exercise effects on physical fitness in older women. Geriatrics \& Gerontology International, 8(4), 265-71. doi: 10.1111/j.1447-0594.2008.00485.x

Church, T.S., Martin, C.K., Thompson, A.M., Earnest, C.P., Mikus, C.R. \& Blair, S.N. (2009). Changes in weight, waist circumference and compensatory responses with different doses of exercise among sedentary, overweight postmenopausal women. PLoS ONE, 4(2), e4515.

Consejo de Europa. (1975). Conferencia de Ministros Europeos responsables del Deporte. Bruselas.

Esparza, F. (1993). Manual de Cineantropometría. Barcelona: Femede.

Faulkner, J.A. (1968). Physiology of swimming and diving. En: Falls, H. (Ed). Exercise physiology. Baltimore: Academic Press.

Gappmaier, E., Lake, W., Nelson, A.G. \& Fisher, A.G. (2006). Aerobic exercise in water versus walking on land: effects on indices of fat reduction and weight loss of obese women. The Journal Of Sports Medicine Physical Fitness, 46(4), 564-9.

García, M. (2006). Veinticinco años de análisis del comportamiento deportivo de la población española (1980-2005). Revista Internacional de Sociología (RIS), 64(44), 15-38.

García, J.M., Navarro, M. y Ruiz, J.A. (1996). Bases teóricas del entrenamiento deportivo. Madrid: Editorial Gymnos.

Giada, F., Vigna, G.B., Vitale, E., Baldo-Enzi, G., Bertaglia, M., Crecca, R. \& Fellin, R. (1995). Effect of age on the response of blood lipids, body composition, and aerobic power to physical conditioning and deconditioning. Metabolism, 44(2), 161-5.

González, E., Núñez, J.M. y Salvador, A. (1997). Efectos de un programa de entrenamiento sobre el estado de ánimo y la ansiedad en mujeres sedentarias. Psicothema, 9 (3), 487-497.

González, J.M., Delgado, M., Contreras, O.R. y Vaquero, M. (2003). Variación antropométrica y de fuerza entre personas de 50 y 70 años practicantes de atletismo y gimnasia de mantenimiento. Revista Española de Geriatría y Gerontología, 38 (2), 79-85.

Greene, N.P., Lambert, B.S., Greene, E.S., Carbuhn, A.F., Green, J.S. \& Crouse, S. F. (2009). Comparative efficacy of water and land treadmill training for overweight or obese adults. Medicine \& Science in Sports \& Exercise, 41(9), 1808-1815. doi: 10.1249/MSS.0b013e3181a23f7f 
Huertas, F., López, A.L., Pablos, A., Colado, J.C., Pablos, C. y Campos, J. (2003). Efectos de un programa de ejercicio físico sobre el bienestar psicológico de mujeres mayores de 55 años. Revista de psicología del deporte, 12(1), 7-26.

Irwin, M.L., Yasui, Y., Ulrich, C.M. Bowen, D., Rudolph, R.E., Schwartz R.S, Yukawa, M., Aiello, E., Potter J.D., McTiernan A. (2003). Effect of exercise on total and intra-abdominal body fat in postmenopausal women: a randomized controlled trial. The Journal of the American Medical Association, 289(3), 323-330.

Jakicic, J.M., Marcus, B.H., Gallagher, K.I., Napolitano, M. \& Lang, W. (2003). Effect of exercise duration and intensity on weight loss in overweight, sedentary women: a randomized trial. The Journal of the American Medical Association, 290(10), 1323-1330.

Jiménez-Beatty, J.E., González, M.D., Rodríguez, M.M., Del Hierro, D. y Martínez, J. (2008). Hábitos de actividad física y demandas de servicios de actividad física de las mujeres adultas. Efdeportes, 12(118).

Kanemaru, A., Arahata, K., Ohta, T., Katoh, T., Tobimatsu, H. \& Horiuchi, T. (2010). The efficacy of home-based muscle training for the elderly osteoporotic women: The effects of daily muscle training on quality of life (QoL). Archives of Gerontology and Geriatrics, 51(2), 169-72.

Lee, R.C., Wang, Z., Heo, M., Ross, R., Janssen, I. \& Heymsfield SB. (2000). Total-body skeletal muscle mass: development and cross-validation of anthropometric prediction models. American Journal Clinical Nutrition, 72(3), 796-803.

López, G., Baena, P.A., Rivas, A., Monteagudo, C., Jiménez, M. y Romero, A. (2005). Comparación de variables dinamométricas y antropométricas en la práctica de actividad física en el medio acuático: Aquaerobic vs Natación. Archivos de Medicina del Deporte: Comunicaciones de biomecánica, 22(110), 524.

Ley, C.J., Lees, B. \& Stevenson, J.C. (1992). Sex- and menopause-associated changes in body-fat distribution. The American Journal of Clinical Nutrition, 55, 950-955.

Marfell-Jones, M., Olds, T., Stewart, A. \& Carter, L. (2006). International standards for anthropometric assessment. South Africa: ISAK Potchefstroom.

Tabernero, B., Villa, J.G., Márquez, S., y García, J. (2000). Cambios en el nivel de condición física, relacionadas con la salid, en mujeres participantes en un programa municipal de baile aeróbico. Apunts: Educación Física yDeportes, 61, 74-79.

Martínez, J., González, M.D., Jiménez-Beatty, J.E., Graupera, J.L., Martín, M., Campos, A. y Del Hierro, D. (2009). Los hábitos de actividad física de las mujeres mayores en España. International Journal of Sport Science, 5(14), 81-93.

Ministerio de Sanidad Política Social e Igualdad. Instituto Nacional de estadística. (2009). Encuesta Europea de Salud en España.

Morilla, M. (2001). Beneficios psicológicos de la actividad física y el deporte. EFdeportes, 7(43).

Nikolai, A.L., Novotny, B.A., Bohnen, C.L., Schleis, K.M. \& Dalleck, L.C. (2009). Cardiovascular and metabolic responses to water aerobics exercise in middle-aged and older adults. Journal of Physical Activity and Health. 6(3), 333-338. 
Nindl, B.C., Harman, E.A., Marx, J.O., Gotshalk, L.A., Frykman, P.N., Lammi. E., Palmer, W.J. \& Kraemer, W.J. (2000). Regional body composition changes in women after 6 months of periodized physical training. Journal of Applied Physiology, 88(6), 2251-2259.

Park, S.K., Park, J.H., Kwon, Y.C., Kim, H.S., Yoon, M.S. \& Park, H.T. (2003). The effect of combined aerobic and resistance exercise training on abdominal fat in obese middle-aged women. Journal of Physiological Anthropology and Applied Human Science, 22(3), 129-135.

Prestes, J., De Lima, C., Frollini, A.B., Donatto, F.F. \& Conte, M. (2009). Comparison of linear and reverse linear periodization effects on maximal strength and body composition. Journal of Strength Conditioning Research, 23(1), 266-274.

Rocha, M. (1975). Peso ósseo do brasileiro de ambos sexos de 17 a 25 anos. Arquivos de Anatomia e Antropología, 1, 445-451.

Castillo, M.J., Ortega, F.B. y Ruiz, J. (2005). Mejora de la forma física como terapia antienvejecimiento. Medicina Clínica, 124 (4), 146-155.

Saavedra, J.M., De la Cruz, E., Escalante, Y. \& Rodríguez F.A. (2007). Influence of a medium-impact aquaerobic program on health-related quality of life and fitness level in healthy adult females. The Journal of Sports Medicine and Physical Fitness, 47(4), 468-474.

Saavedra, J.M., Escalante, Y., Pino, J., De la Cruz, E. y Rodríguez, F.A. (2006). Aplicación de un programa de aquaeróbic de muy corta duración (4 semanas) para la mejora de la condición física saludable en mujeres jóvenes sanas. Apunts Educació Física i esport, 86, 14-21.

Shimamoto, H., Adachi, Y., Takahashi, M. \& Tanaka, K. (1998). Low impact aerobic dance as a useful exercise mode for reducing body mass in mildly obese middle-aged women. Applied Human Science, 17(3), 109-114. doi: 10.1359/jbmr.2000.15.12.2495

Winters, K.M. \& Snow, C.M. (2000). Detraining reverses positive effects of exercise on the musculoskeletal system in premenopausal women. Journal of Bone and Mineral Research ${ }_{2} 15$ (12), 2495-2503.

Fecha de recepción: 27 de enero del 2011.

Fecha de aceptación: 21 de julio del 2011.

Fecha de publicación: 31 de julio del 2011. 